\title{
A vision specific functional index for use in patients with age related macular degeneration
}

\author{
P M Hart, U Chakravarthy, M R Stevenson, J Q Jamison
}

\begin{abstract}
Aim-To develop and evaluate a new vision specific functional index for use in individuals with age related macular degeneration (AMD).

Methods-Following consultation with patients with AMD and healthcare professionals, a questionnaire entitled the Daily Living Tasks Dependent on Vision (DLTV) was constructed. It was administered by interview to three separate groups of individuals aged 50 years or older: people with AMD, people with cataract, and people with no visual disability. The relations between DLTV, distance visual acuity, and disorder were examined using Pearson's product moment correlation coefficients, stepwise regression, and principal component analysis.

Results-There was a positive correlation between DLTV items and distance visual acuity in the better eye. Principal component analysis showed that the DLTV has a major single dimension within it. This first principal component accounted for $59 \%$ of the variation and correlated well with distance visual acuity in the better eye. Other components were found, one of which correlated with the difference in vision in the two eyes and one which featured in the differentiation of AMD subjects from individuals with cataracts.
\end{abstract}

Conclusions-The DLTV provided information on visual impairment in patients over and above that obtained from a measure of visual acuity. It also showed that patients with AMD experience greater difficulty with daily living tasks for any given level of acuity than do patients with cataract.

(Br f Ophthalmol 1999;83:1115-1120)

Ophthalmology and

Vision Science,

Queen's University of

Belfast

P M Hart

U Chakravarthy

Health and Health Care Research Unit, Queen's University of Belfast

M R Stevenson

J Q Jamison

Correspondence to: P M Hart, Ophthalmology and Vision Science, Royal Victoria Hospital, Belfast BT12 6BA.

Accepted for publication 18 May 1999
Functioning Index (VFI), the Visual Function 14 or 12 item scale (VF14 or VF12), ${ }^{67}$ and the Activities of Daily Vision Scale
(ADVS). ${ }^{8}$ At present, instruments to systematically document the patient's perception have only occasionally been used as outcome measures in assessing the effects of treatment in other ocular diseases. ${ }^{9-11}$ In each case a disease specific questionnaire was constructed, as it is recognised that a questionnaire devised with one disorder in mind may well be inappropriate if used in patients with a different disorder. ${ }^{9-11}$ At the inception of this study no suitable instrument was available for application in patients with age related macular degeneration.

Although it is standard ophthalmic practice to assess "vision" by recording the patient's distance and near visual acuity, it is now widely recognised that these traditionally accepted measurements do not reflect visual function comprehensively. ${ }^{12}{ }^{13}$ Some reasons suggested to explain this discrepancy include the facts that (a) Snellen acuity only measures function within the central $1^{0}$ to $2^{0}$ of the visual field, ${ }^{14}$ (b) this measurement is in monochrome and is at $100 \%$ contrast, unlike the real world, ${ }^{15}$ and (c) psychophysical testing has shown the necessity for considerable visual reserve - that is, one may be able to visually resolve words of a given character size, but can read text fluently only when print size is considerably larger. ${ }^{16}{ }^{17}$ Although essential to record, the results of tests such as distance acuity, near acuity, or contrast sensitivity are limited in their ability to quantify visual function or its corollary, visual dysfunction. In practice, most clinicians attempt to overcome this problem by ad hoc history taking, but it is apparent that a more systematic means of collecting information would facilitate reproducible and accurate data recording and aid in the monitoring of outcomes.

As previously stated most visual function questionnaires already in existence were developed primarily to detect the changes in visual function which follow cataract extraction..$^{5-8}$ In this situation near perfect visual restoration may be expected and many of the questions in these instruments were designed to assess the remediable disabilities typically found in patients suffering from cataract. ${ }^{8}$ By comparison, patients with age related macular degeneration (AMD) exhibit a visual disability which is more complex, often more profound, and is qualitatively different from that caused by cataract. ${ }^{18} 19$ As such, their function might not be assessed adequately using existing instruments. It was felt that in addition to reaching a floor effect quickly, some patients would be depressed by their repeatedly low and usually decreasing scores. In order to 
Table 1 The complete questionnaire and the scoring system for the DLTV (Daily living tasks dependent on vision)

\begin{tabular}{|c|c|c|c|c|c|}
\hline \multicolumn{6}{|c|}{ How much difficulty do you have } \\
\hline & & $\begin{array}{l}\text { No } \\
\text { difficulty }\end{array}$ & $\begin{array}{l}\text { A little } \\
\text { difficulty }\end{array}$ & $\begin{array}{l}\text { A lot of } \\
\text { difficulty }\end{array}$ & $\begin{array}{l}\text { Cannot } \\
\text { see to do }\end{array}$ \\
\hline 1 & Distinguishing a person's features across the room & 4 & 3 & 2 & 1 \\
\hline 2 & Noticing objects off to either side & 4 & 3 & 2 & 1 \\
\hline 3 & Watching TV programmes & 4 & 3 & 2 & 1 \\
\hline 4 & Seeing steps and using them & 4 & 3 & 2 & 1 \\
\hline 5 & Enjoying the scenery if out for a drive & 4 & 3 & 2 & 1 \\
\hline 6 & Reading road signs/street names & 4 & 3 & 2 & 1 \\
\hline 7 & Distinguishing a person's features across the street & 4 & 3 & 2 & 1 \\
\hline 8 & Recognising seasonal changes in the garden & 4 & 3 & 2 & 1 \\
\hline 9 & Distinguishing a person's features at arm's length & 4 & 3 & 2 & 1 \\
\hline 10 & Pouring yourself a drink & 4 & 3 & 2 & 1 \\
\hline 11 & Cutting up food on your plate & 4 & 3 & 2 & 1 \\
\hline 12 & Cutting your finger nails & 4 & 3 & 2 & 1 \\
\hline 13 & Using kitchen appliances & 4 & 3 & 2 & 1 \\
\hline 14 & Adjusting to darkness after being in the light & 4 & 3 & 2 & 1 \\
\hline 15 & Adjusting to the light after being in the dark & 4 & 3 & 2 & 1 \\
\hline \multicolumn{6}{|c|}{ How confident do you feel in your ability to walk around } \\
\hline & & Extremely & Somewhat & Barely & Not at all \\
\hline 16 & In your immediate neighbourhood & 4 & 3 & 2 & 1 \\
\hline 17 & Outside your immediate neighbourhood & 4 & 3 & 2 & 1 \\
\hline \multicolumn{6}{|c|}{ With your near glasses on how much difficulty do you have } \\
\hline & & $\begin{array}{l}\text { No } \\
\text { difficulty }\end{array}$ & $\begin{array}{l}\text { A little } \\
\text { difficulty }\end{array}$ & $\begin{array}{l}\text { A lot of } \\
\text { difficulty }\end{array}$ & $\begin{array}{l}\text { Cannot } \\
\text { see to do }\end{array}$ \\
\hline 18 & Reading normal sized newspaper print & 4 & 3 & 2 & 1 \\
\hline 19 & Reading newspaper headlines & 4 & 3 & 2 & 1 \\
\hline 20 & Reading correspondence-eg, bills, letters, cards & 4 & 3 & 2 & 1 \\
\hline 21 & Signing documents (cheques, pension book) & 4 & 3 & 2 & 1 \\
\hline 22 & Identifying money from purse or wallet & 4 & 3 & 2 & 1 \\
\hline \multicolumn{6}{|c|}{ How would you rate } \\
\hline & & Excellent & Good & Fair & Poor \\
\hline 23 & Your overall distance vision & 4 & 3 & 2 & 1 \\
\hline 24 & Your overall near vision (ie, for close work) & 4 & 3 & 2 & 1 \\
\hline
\end{tabular}

Note scores from 23 and 24 are not included in summary DLTV score.

widen the applicability of visual function questionnaires, a new instrument was devised to examine aspects of visual function potentially affected in people with AMD. This instrument was then piloted in three groups of subjects over 55 years of age. Two groups suffered from a visual disorder (AMD and cataract) and the third group comprised individuals with no visual loss (controls).

\section{Methods}

Patients with AMD were asked to list daily living activities with which they had difficulty because of their visual disability. Sex specific activities, such as applying make up and shaving, were excluded as it was often not possible to equate them in terms of skills required (for example, men volunteered the fact that they used an electric shaver by touch). Activities of a particularly individual nature were also excluded because of their limited applicability across the population of interest. The remaining activities were identified as those with which many AMD patients commonly had difficulty. This list was then circulated to healthcare professionals associated with the care of visually handicapped people, with an invitation to suggest other questions from their experience. A final list of tasks identified both by patients and health professionals was then drawn up and questions devised to address the relevant problems. Some questions were added about activities, which would not normally cause any difficulty in AMD patients, to ensure an adequate breadth of possible responses.
The activities fell into several broad categories reflecting various aspects of visual function which included far distance, intermediate and near vision, binocularity, field of vision, light and dark adaptation, and contrast sensitivity. The questions comprising the instrument, named the Daily Living Tasks Dependent on Vision (DLTV) are shown in Table 1. Respondents were asked to score each of these to reflect the degree of difficulty with which they could accomplish the task concerned. Both the syntax and the scoring system were similar to those used in previous studies examining the effect of cataract on visual function. ${ }^{6}{ }^{8}$ In the present study, the question posed to the patient was "how much difficulty do you have" followed by the particular activity. The possible responses with their scores are: $4=$ no difficulty; $3=$ a little difficulty; 2 = a lot of difficulty; 1 = vision prevents from doing.

If the activity was not performed for a reason unrelated to vision this was noted and the question deemed unanswered. A summary DLTV score can be obtained by adding the scores given for each item, dividing by the number of questions answered and expressing this on a scale of $0-100$.

Some instruments have used a form of questioning which invites respondents to grade their perceived level of overall visual function (global self rating of vision) rather than rate the difficulty in performing specific tasks. ${ }^{67}$ We therefore added two such questions (items 23 and 24) on the subject's overall impression of their distance and near visual function using a similar ordinal scale from 1-4 indicating poor, fair, good, or excellent (Table 1). The responses to items 23 and 24 would not be included in the DLTV summary score.

\section{SUBJECTS}

A pre-pilot exercise was conducted to establish variation in visual acuity and individual DLTV items across subjects. This suggested that a sample size of 25-35 per group was required.

Subjects were obtained in four ways: (a) elderly patients attending a macular degeneration clinic; (b) patients about to undergo cataract surgery; (c) patients attending a general practitioner's geriatric screening unit; (d) elderly patients attending a local hospital's rehabilitation unit.

All subjects were over 55 years of age. Subjects recruited under (c) and (d) above formed the control group and were required to have a distance visual acuity of $6 / 12$ or better in each eye, have no visual complaints, and be able to read a daily newspaper with their current reading spectacles.

The method of sampling was essentially "quota" on a first come first served basis. One hundred and eight eligible subjects were identified and all consented to interview. The DLTV was administered in a personal interview by any one of three people. The questionnaires from five subjects were rejected because of large amounts of missing 
Table 2. Average logMAR visual acuities and the sex distribution of the subjects in the three disorder groups. Age, sex, and visual acuity distribution by condition

\begin{tabular}{llllll}
\hline Condition & Mean age & $\begin{array}{l}\text { Mean of average } \\
\text { visual acuity }(S D)\end{array}$ & Female & Male & Total number \\
\hline Control & 76.1 & $0.17(0.16)$ & 21 & 11 & 32 \\
Cataract & 73.7 & $0.74(0.58)$ & 28 & 9 & 37 \\
AMD & 74.0 & $0.93(0.56)$ & 22 & 12 & 34 \\
\hline
\end{tabular}

data. The 103 subjects remaining were distributed as 32 controls, 37 cataract, and 34 AMD patients. All subjects had their visual acuity measured on the Snellen eye chart with their current spectacle correction if appropriate.

\section{STATISTICAL ANALYSIS}

Interobserver differences were examined using one way analysis of variance since different interviewers had recorded responses on different patients.

It is recognised that there is no gold standard measure of visual function. As distance visual acuity (DVA) is the most widely used and valued measure of visual function and was available on all the subjects interviewed in this study, an initial analysis was performed to seek the correlation between DVA and the DLTV. All Snellen acuity results were converted into the $\log _{10}$ of the angle of resolution - that is, logMAR equivalents, to facilitate analysis (logMAR acuity progresses geometrically with a fall in acuity by 3 lines between any two points on the scale representing a doubling of the visual angle). A $\log$ MAR acuity of 0 equates to a Snellen acuity of $6 / 6$ (normal vision) and a $\log$ MAR acuity of 1 is Snellen 6/60. Thus, an increase in the acuity score indicates worsening of visual function.

We attempted to find the measure of acuity which would most accurately predict a person's perceived ability to perform visually dependent tasks by examining the product moment correlations between the individual

Table 3 Correlation between the DLTV items and each of minVA, maxVA, and avgVA for all three disorder groups. Negative correlations are seen because on the logMAR scale better vision is represented by smaller numbers

\begin{tabular}{llll}
\hline Item & $\begin{array}{l}\text { Acuity in } \\
\text { better eye }\end{array}$ & $\begin{array}{l}\text { Acuity in } \\
\text { worse eye }\end{array}$ & $\begin{array}{l}\text { Average } \\
\text { acuity }\end{array}$ \\
\hline Distinguishing faces across a room & -0.64 & -0.38 & -0.55 \\
Seeing objects off to one side & -0.21 & -0.28 & -0.29 \\
Watching TV & -0.56 & -0.50 & -0.60 \\
Seeing and using steps & -0.37 & -0.56 & -0.57 \\
Enjoying scenery & -0.61 & -0.46 & -0.60 \\
Reading road signs & -0.71 & -0.55 & -0.71 \\
Distinguishing faces across the street & -0.65 & -0.60 & -0.71 \\
Recognising seasonal changes & -0.59 & -0.44 & -0.58 \\
Distinguishing faces at arm's length & -0.51 & -0.34 & -0.46 \\
Pouring oneself a drink & -0.46 & -0.41 & -0.50 \\
Cutting food on a plate & -0.36 & -0.30 & -0.37 \\
Cutting finger nails & -0.64 & -0.42 & -0.58 \\
Using kitchen appliances & -0.41 & -0.37 & -0.44 \\
Adjusting to darkness & -0.39 & -0.37 & -0.44 \\
Adusting to light & -0.26 & -0.44 & -0.44 \\
Confidence in immediate neighbourhood & -0.22 & -0.46 & -0.43 \\
Confidence outside neighbourhood & -0.44 & -0.52 & -0.57 \\
Read normal sized newsprint & -0.68 & -0.58 & -0.72 \\
Read newspaper headlines & -0.66 & -0.47 & -0.63 \\
Read correspondence & -0.68 & -0.59 & -0.73 \\
Signing documents & -0.68 & -0.46 & -0.63 \\
Identifying money & -0.68 & -0.51 & -0.66 \\
Self rating of overall distance vision & -0.56 & -0.54 & -0.63 \\
Self rating of overall near vision & -0.55 & -0.48 & -0.58 \\
\hline
\end{tabular}

Significance levels: correlation coefficient $r=0.19{ }^{\star} \mathrm{p}<0.05, r=0.25{ }^{\star} \mathrm{p}<0.01, r=0.32$ * $\mathrm{p}<0.001$. items in the DLTV and each of the three measures of distance visual acuity-namely, acuity in the better eye, acuity in the worse eye, and average acuity in the two eyes. As the correlations between acuity in the worse eye and the DLTV were significant, the impact of this eye on a patient's ability to perform any task was examined by including the variable "difference in visual acuity" (as explained in results).

Stepwise regression was used to determine how acuity in the better eye, the difference in acuity between the two eyes, and the presence of a specific visual disorder (AMD or cataract) influenced responses on the DLTV. The resulting coefficients of regression indicate the degree of relation between performance on the DLTV and each independent variable after the effects of all other variables have been accounted for. Only regression coefficients which were significant at $p<0.05$ are presented. We also examined how well the items of global self rating of vision (items 23 and 24) correlated with measures of acuity using Pearson's product moment correlation coefficient.

Finally, principal component analysis was performed on the results from the entire study population, to examine the overall performance of the DLTV. This is a mathematical tool to identify independent factors (components) within a multivariate set of data. We expected distance visual acuity to have a major influence on the DLTV scores but assumed that the DLTV would provide more information than would be obtained from measuring distance visual acuity alone.

\section{Results}

GENERAL OBSERVATIONS ON QUESTIONNAIRE ADMINISTRATION

The DLTV was found to be acceptable to patients and the questionnaires were generally completed within 6-10 minutes. There was no significant difference in DLTV responses obtained by the three different interviewers ( $F$ $=1.56$ on 2 and $98 \mathrm{df}: \mathrm{p}>0.05$ ).

ANALYSIS OF THE DLTV

The age and sex distribution, and the average $\log$ MAR visual acuities of the subjects in the three groups are shown in Table 2. There was no significant difference in age distribution between the three groups; however, mean visual acuities were significantly different in the three groups with worst visual acuity seen in patients with AMD and the best in controls.

The correlations between the individual DLTV items and (i) distance visual acuity in the better eye, (ii) in the worse eye, and (iii) the average of both eyes are shown in Table 3 . Since better vision is represented as a lower visual acuity score the correlations are negative numbers. All correlation coefficients were significant $(p<0.05)$ and in the direction anticipated. Moderate correlations were seen with most items. There was a tendency towards higher correlation coefficients between DLTV items and both "acuity in the better eye" and 
Table 4 Results of a series of stepwise regression analysis looking at DLTV score as a function of distance visual acuity (using two measures - distance VA in the better eye and difference in VA between eyes) and the disorder (cataract, AMD, or neither)

\begin{tabular}{|c|c|c|c|c|c|c|c|}
\hline \multirow[b]{2}{*}{ Item } & \multirow[b]{2}{*}{$R^{2}$} & \multirow[b]{2}{*}{$S E$} & \multirow[b]{2}{*}{ Constant } & \multicolumn{4}{|c|}{ Regression coefficients } \\
\hline & & & & $\begin{array}{l}\text { Acuity in } \\
\text { better eye }\end{array}$ & $\begin{array}{l}\text { Acuity } \\
\text { difference }\end{array}$ & $\begin{array}{l}\text { AMD } \\
\text { present }\end{array}$ & $\begin{array}{l}\text { Cataract } \\
\text { present }\end{array}$ \\
\hline Distinguish faces across a room & 0.450 & 0.71 & 4.05 & -1.43 & & -0.50 & \\
\hline Seeing objects off to the side & 0.068 & 0.59 & 3.95 & -0.40 & -0.22 & & \\
\hline Watching TV & 0.378 & 0.62 & 4.10 & -1.30 & -0.39 & & \\
\hline Seeing and using steps & 0.318 & 0.60 & 4.07 & -0.87 & -0.56 & & \\
\hline Enjoying scenery & 0.403 & 0.59 & 4.25 & -1.34 & -0.28 & & \\
\hline Reading road signs & 0.587 & 0.68 & 4.13 & -1.95 & -0.37 & -0.38 & \\
\hline Distinguishing faces across the street & 0.600 & 0.73 & 4.12 & -1.79 & -0.50 & -0.75 & \\
\hline Recognising seasonal changes & 0.381 & 0.50 & 4.23 & -1.10 & -0.22 & & \\
\hline Distinguishing faces at arm's length & 0.303 & 0.55 & 4.15 & -0.72 & & -0.37 & \\
\hline Pouring oneself a drink & 0.419 & 0.46 & 4.05 & -0.43 & & -0.65 & \\
\hline Cutting food on a plate & 0.176 & 0.52 & 4.01 & -0.40 & & -0.33 & \\
\hline Cutting finger nails & 0.438 & 0.69 & 4.06 & -1.41 & & -0.40 & \\
\hline Using kitchen appliances & 0.320 & 0.56 & 4.01 & -0.44 & & -0.63 & \\
\hline Adjusting to darkness & 0.193 & 0.72 & 3.77 & -1.01 & -0.32 & & \\
\hline Adjusting to light & 0.227 & 0.69 & 3.82 & -0.64 & -0.43 & & -0.36 \\
\hline Confidence in immediate neighbourhood & 0.191 & 0.70 & 4.11 & -0.61 & -0.54 & & \\
\hline Confidence outside neighbourhood & 0.310 & 0.93 & 4.02 & -1.58 & -0.71 & & \\
\hline Read normal sized newsprint & 0.662 & 0.71 & 4.24 & -1.88 & -0.42 & -0.96 & \\
\hline Read newspaper headlines & 0.462 & 0.61 & 4.33 & -1.56 & -0.29 & & \\
\hline Read correspondence & 0.633 & 0.71 & 4.37 & -1.88 & -0.46 & -0.77 & \\
\hline Signing documents & 0.537 & 0.65 & 4.20 & -1.56 & & -0.64 & \\
\hline Identifying money & 0.550 & 0.58 & 4.26 & -1.42 & -0.23 & -0.47 & \\
\hline Self rating of overall distance vision & 0.404 & 0.76 & 3.32 & -1.70 & -0.55 & & \\
\hline Self rating of overall near vision & 0.353 & 0.74 & 3.06 & -1.46 & -0.39 & & \\
\hline
\end{tabular}

$R^{2}=$ coefficient of determination; $\mathrm{SE}=$ standard error of the prediction.

"the average acuity of the individual's two eyes". The overall analysis confirmed that the measure of vision which correlated best with DLTV scores was distance visual acuity in the better eye.

Three items-namely, seeing and using steps, adjusting to light after being in the dark, and confidence when within one's immediate neighbourhood, correlated more strongly with acuity in the worse eye and with average acuity than with acuity in the better eye.

There were moderate correlations found between scores of global self rating of vision (GSR), for near and distance, and all measures of visual acuity. Average VA showed the strongest correlations-namely, 0.63 with GSR distance and 0.58 with GSR near.

Stepwise regression analysis was used to examine the relation between DLTV scores, vision in the better eye, the difference in acuity between the two eyes, and the disorder. Difference in VA was used as this computed measure reflects the additional influence of the worse eye but is not correlated with visual acuity in the better eye $(r=-0.14: \mathrm{p}>0.05)$. This analysis showed that tasks which tested the subject's ability to visually discriminate detail had larger coefficients of determination $\left(R^{2}\right)$. This indicates that a greater proportion of variability in DLTV scores was accounted for by the combination of visual acuity in the better eye, difference in visual acuity between the two eyes, and the disease status (Table 4). Other items-for example, noticing objects off to one side which examined factors such as navigational vision had lower coefficients.

Where a regression coefficient is missing in Table 4, it failed to reach significance at the level $\mathrm{p}<0.05$. In this case the impact of the relevant independent variable can be taken as zero. People suffering from AMD appeared to fare worse on certain tasks than would have been predicted on the basis of their acuity alone. This can be seen from Table 4, column 7 (AMD present), where on 12 items of the DLTV, AMD patients are seen to be differentially disadvantaged when compared with either the cataract or the control group. That aspect of visual function differentially affected in cataract patients appeared to be difficulty in adjusting to light after having been in the dark (column 8).

Principal component analysis showed that the first principal component was formed by a weighted average of most items measured within the DLTV. This component correlated well with the measures of visual acuity, particularly acuity in the better eye, and it explained $59 \%$ of total variation suggesting that, although not unidimensional, the DLTV has a major single dimension within it. The second principal component explained $7 \%$ of the variation and it correlated with the difference in visual acuity between the two eyes. The third principal component accounted for $5 \%$ of the variation and essentially differentiated between the two disease statesthat is, cataract and AMD.

\section{Discussion}

The DLTV was devised bearing in mind the profound central visual loss which occurs in patients with AMD, and it has provided further evidence that such individuals experience serious disability when coping with vision related tasks around their home and in their neighbourhood. Not surprisingly, the scores for DLTV items correlated most strongly with acuity in the better eye. This is in accordance with the findings of other vision specific questionnaires. ${ }^{6}$ However, a correlation was also found to be present between DLTV scores and acuity in the worse eye, suggesting a definite contribution from the latter to visual 
function. Although it was not the remit of this study to examine the extent of the contribution of the weaker eye to visual function, our findings are in broad agreement with those of other investigators who have demonstrated improvements in quality of life following cataract surgery in the second eye. ${ }^{20}$ It is interesting to note that simple global self rating for near and distance, correlating most strongly as they did with the average vision in the two eyes, also recognised the contribution of two eyes to patients' perception of their visual function.

Within the DLTV certain activities were detected with which AMD patients experienced disproportionate difficulty compared with the cataract group when visual acuity was held constant. The tasks concerned were those which involved ability to distinguish facial characteristics, reading correspondence or newspapers, and pouring oneself a drink. The visual functions of contrast sensitivity, visual search, processing speed, depth perception, and vernier acuity all play important roles in the completion of such tasks. The greater difficulty experienced by AMD patients may reflect the gravity of the underlying pathology of their condition and indicate the inability of the macular photoreceptors to process visual stimuli in this group. Since many of the other visual problems experienced by cataract patients are a reflection of an overall reduction in the visual stimulus rather than an intrinsic fault in the photoreceptors, it is perhaps understandable that when visual acuity is accounted for, patients with cataract fare very similarly to controls on the DLTV. As identified by previous studies, ${ }^{68}$ however, people with cataract appeared to have a disproportionate amount of trouble adjusting to bright light conditions after having been in the dark, indicative of significant glare disability.

An overall description of the performance of the DLTV was provided by principal component analysis. This showed, not unexpectedly, that the mass of questions within the questionnaire was highly correlated with distance visual acuity in the better eye. However, the DLTV reflected substantially more than this in that approximately $40 \%$ of the variation seen in DLTV scores could not be explained on the basis of distance visual acuity in the better eye alone. The second principal component explained $7 \%$ of the variation and correlated with the difference in acuity between two eyes, again-that is, there was recognition of a contribution from the second eye. The third principal component identified a difference between patients suffering from cataract and patients suffering from AMD. Subsequent components accounted for ever decreasing amounts of the total variation. For practical purposes we did not proceed beyond the third principal component which accounted for 5\% of the variation in this analysis. Future work with greater numbers could attempt to identify other variables which are being registered by the DLTV as contributing to difficulties in visual function. The multidimensional properties of this instrument satisfy the original aim of the study - that is, to provide information on patients with AMD over and above that obtained from a measure of distance visual acuity. The analysis suggests that the instrument described in the present study would be responsive to changes in many aspects of "vision", such as binocular function, factors relating to field of vision, light/dark adaptation, glare disability, depth perception, and psychometric associations-for example, confidence in getting around.

This study did not address the test-retest reliability or sensitivity of the instrument to change over time. Subsequent to this study, we have allowed the questionnaire to be self administered, employed an assisted self administration methodology (where a friend or relative performs the role of reading the questions and recording responses with strict instruction neither to prompt nor to amend the given answers), and used telephone mediated interviews. All of the aforementioned are currently undergoing investigation.

The present study has shown that the DLTV is a useful tool in the evaluation of an individual's visual status and has highlighted the difficulties encountered by patients even in early age related macular disease. More recently the NEIVFQ (National Eye Institute Visual Function Questionnaire) has been developed in the USA, potentially for use in a wide range of visual disorders. It is yet to be established whether the content area identified for this questionnaire will have validity for people with severe vision loss. ${ }^{21}$ Comparison of the DLTV and NEIVFQ is a component of the ongoing evaluation of the DLTV in a larger cohort of individuals with age related macular degeneration.

The support of the Medical Research Council is gratefully acknowledged. The authors are also grateful to Mrs P McEvoy for her help with conducting the interviews.

1 Read JL, Quinn RJ, Hoefer MA. Measuring overall health: an evaluation of three important approaches. 7 Chron Dis an evaluation of thre

2 Fitzpatrick R, Fletcher A, Gore S, et al. Quality of life measures in health care. I. Applications and issues in assessment. BMF 1992;305:1074-7.

3 Ebbs SR, Fallowfield LJ, Fraser CA, et al. Treatment outcomes and quality of life. Int F Technol Assess Health Care 1989;5:391-400

4 Patrick DL, Deyo RA. Generic and disease-specific measures in assessing health status and quality of life. Med Care 1989;27:S217-31

5 Bernth-Petersen P. Visual functioning in cataract patients. Methods of measuring and results. Acta Ophthalmol 1981; 59:198-205.

6 Steinberg EP, Tielsch JM, Schein OD. The VF 14: an index of functional impairment in patients with cataract. Arch Ophthalmol 1994;112:630-8.

7 Tielsch JM, Steinberg EP, Cassard SD, et al. Pre-operative functional expectations and post-operative outcomes functional expectations and post-operative outcomes Ophthalmol 1995;113:1312-18.

8 Mangione CM, Phillips RS, Seddon JM, et al. Development of the activities of daily vision scale: a measure of visual functional status. Med Care 1992;30:1111-26.

9 Lee BL, Gutierrez P,Wilson R, et al. The glaucoma symptom scale. Arch Ophthalmol 1998;116:861-6.

10 Wu AW, Coleston LC, Holbrook J, et al. Measuring visual function and quality of life in patients with cytomegalic retinitis. Arch Ophthalmol 1996;114:841-7.

11 BattuVK, Meyer DR, Wobig JL. Improvement in subjective visual function and quality of life outcome measures after blepharoptosis surgery. Am $\mathcal{f}$ Ophthalmol 1996;121:677-86.

12 Cheng AS, Vingrys AJ. Visual losses in early age-related maculopathy. Optom Vis Sci 1995;70:89-96.

13 Elliot DB, Hurst MA, Wetherill JR. Comparing clinical tests of visual function in cataract with the patient's percieved of visual function in cataract with the

14 Wetherill JR. Visual acuity assessment. Eye 1993;7:26-8. 
15 Reeves BC, Wood JM, Hill AR. Reliability of high and low contrast letter charts. Ophthalmic Physiol Opt 1993;13:17-26.

16 Legge GE. The Glenn A Fry Award Lecture. Three perspectives on low vision reading. Optom Vis Sci 1991;63 $763-9$

17 Rumney NJ. Using visual thresholds to establish low vision performance. Ophthalmic Physiol Opt 1995;15:S18-24.

18 Alexander MF, Maguire MG, Lietman TM, et al. Assessment of visual function in patients with age-related macular degeneration. Arch Ophthalmol 1988;106:1543-7.
19 Brown B, Zadnik Z, Bailey IL, et al. Effect of luminance, contrast and eccentricity on visual acuity in senile macular

20 Javitt JC, Brenner MH, Curbow B, et al. Outcomes of cataract surgery. Improvement in visual acuity and subjective
visual function after surgery in the first, second and both eyes. Arch Ophthalmol 1993;111:686-91.

21 Mangione CM, Berry S, Spritzer K, et al. Identifying the content area for the 51-item National Eye Institute visual function questionnaire. Arch Ophthalmol 1998;116:227-33. 